\title{
miR-31-NUMB Cascade Modulates Monocarboxylate Transporters to Increase Oncogenicity and Lactate Production of Oral Carcinoma Cells
}

\author{
Chung-Hsien Chou ${ }^{1}$, Chun-Yu Fan Chiang ${ }^{1}$, Cheng-Chieh Yang ${ }^{1,2,3}{ }^{\mathbb{D}}$, Ying-Chieh Liu ${ }^{1}$, Sih-Rou Chang ${ }^{1}$, \\ Kuo-Wei Chang $1,2,3, * \mathbb{D}$ and Shu-Chun Lin $1,2,3, * \mathbb{D}$
}

1 Institute of Oral Biology, College of Dentistry, National Yang Ming Chiao Tung University, Taipei 112, Taiwan; michaelchou0806@gmail.com (C.-H.C.); taroislove@hotmail.com (C.-Y.F.C.); ccyang@ym.edu.tw (C.-C.Y.); yingchieh12@gmail.com (Y.-C.L.); s4103052122@gmail.com (S.-R.C.)

2 Department of Dentistry, College of Dentistry, National Yang Ming Chiao Tung University, Taipei 112, Taiwan

3 Department of Stomatology, Taipei Veterans General Hospital, Taipei 112, Taiwan

* Correspondence: ckcw@nycu.edu.tw (K.-W.C.); shuchun@nycu.edu.tw (S.-C.L.)

\section{check for}

updates

Citation: Chou, C.-H.; Chiang, C.-Y.F.; Yang, C.-C.; Liu, Y.-C.; Chang,

S.-R.; Chang, K.-W.; Lin, S.-C. miR-31-NUMB Cascade Modulates Monocarboxylate Transporters to Increase Oncogenicity and Lactate Production of Oral Carcinoma Cells. Int. J. Mol. Sci. 2021, 22, 11731. https://doi.org/10.3390/ ijms222111731

Academic Editor: Eric Parkinson

Received: 1 October 2021

Accepted: 26 October 2021

Published: 29 October 2021

Publisher's Note: MDPI stays neutral with regard to jurisdictional claims in published maps and institutional affiliations.

Copyright: (c) 2021 by the authors. Licensee MDPI, Basel, Switzerland. This article is an open access article distributed under the terms and conditions of the Creative Commons Attribution (CC BY) license (https:/ creativecommons.org/licenses/by/ $4.0 /)$.
Abstract: Oral squamous cell carcinoma (OSCC) is among the leading causes of cancer-associated death worldwide. miR-31 is an oncogenic miRNA in OSCC. NUMB is an adaptor protein capable of suppressing malignant transformation. Disruption of the miR-31-NUMB regulatory axis has been demonstrated in malignancies. Mitochondrial dysfunction and adaptation to glycolytic respiration are frequent events in malignancies. Monocarboxylate transporters (MCTs) function to facilitate lactate flux in highly glycolytic cells. Upregulation of MCT1 and MCT4 has been shown to be a prognostic factor of OSCC. Here, we reported that miR-31-NUMB can modulate glycolysis in OSCC. Using the CRISPR/Cas9 gene editing strategy, we identified increases in oncogenic phenotypes, MCT1 and MCT4 expression, lactate production, and glycolytic respiration in NUMB-deleted OSCC subclones. Transfection of the Numb1 or Numb4 isoform reversed the oncogenic induction elicited by NUMB deletion. This study also showed, for the first time, that NUMB4 binds MCT1 and MCT4 and that this binding increases their ubiquitination, which may decrease their abundance in cell lysates. The disruptions in oncogenicity and metabolism associated with miR-31 deletion and NUMB deletion were partially rescued by MCT1/MCT4 expression or knockdown. This study demonstrated that NUMB is a novel binding partner of MCT1 and MCT4 and that the miR-31-NUMB-MCT1/MCT4 regulatory cascade is present in oral carcinoma.

Keywords: CRISPR; lactate; MCT1; MCT4; miR-31; NUMB

\section{Introduction}

Oral squamous cell carcinoma (OSCC) is one of the most prevalent head and neck SCCs (HNSCCs) worldwide. miRNAs are noncoding RNAs that play various important roles during pathogenesis by specifically targeting transcripts and regulating their expression levels [1,2]. $m i R-31$ has been shown to be an oncogenic miRNA upregulated in OSCC that modulates hypoxia, DNA repair, stemness, and various metabolic pathways [2-8]. miR-31 targets SIRT3 [7], ACOX1 [8], FIH [9,10], and SDHA [11] to disrupt metabolic homeostasis in malignancies. However, to develop therapeutic strategies, the functions of miRNAs in regulating the metabolism of OSCC must be elucidated.

NUMB is an adaptor protein that plays multifaceted roles in modulating cell functions, including neurogenesis, symmetric cell division in the stemness process, epithelialmesenchymal transition, and oncogenesis [12-15]. NUMB participates in the process of ubiquitin degradation by binding with ubiquitin ligase Itch to breakdown Notch or Gli [16-18]. In addition, NUMB also interacts with p53, MDM2, and PTEN to modulate their activity in the neoplastic process [14,16,19-22]. miR-31 targets NUMB to enhance oncogenicity in OSCC and colorectal carcinoma $[23,24]$. In addition, miR-96, miR-146a, miR-182, 
and $m i R-545$ also target $N U M B$ to augment oncogenicity $[25,26]$. The suppressor roles of $N U M B$ against OSCC have been identified in previous studies $[14,23]$, but its downstream effectors remain to be elucidated. The distinct functions mediated by six NUMB isoforms resulting from alternative splicing have been actively investigated [12,27]. We discovered that the NUMB isoforms Numb1 to Numb4 were capable of suppressing OSCC pathogenesis [23]. Interestingly, a recent study revealed that Numb1, but not Numb4, binds the mGluR5 $\alpha$ glutamate receptor to inhibit its internalization in 293T cells [28]. Whether NUMB variants differentially modulate downstream effectors requires further investigation.

Metabolic symbiosis between oxidative and glycolytic cells is crucial for sustained tumor growth and metastasis. Crosstalk between SLC2A glucose transporter (GLUT) family members and SCL16A monocarboxylate transporter (MCT) family members is responsible for substrate transport in metabolic processing [29,30]. Like in many other types of malignancies, $\mathrm{H}^{+}$-linked lactate transporters MCT1 (SLC16A1) and MCT4 (SLC16A3), which shuttle lactate along the $\mathrm{H}^{+}$gradients, have been relatively well investigated compared with other MCTs in HNSCC [29,31-33]. Upregulation of MCT1 and/or MCT4 expression has also been shown to be a prognostic marker of HNSCC [34,35]. Complex mechanisms including transcriptional modulation, miRNA targeting, post-translational modifications, and others may be involved in regulating the expression and functions of MCT1/MCT4 [30]. The present study provided novel clues demonstrating that NUMB binds MCT1/MCT4 to induce MCT1/MCT4 degradation, decrease oncogenicity, and increase glycolytic metabolism in OSCC cells. The miR-31-NUMB-MCT1/MCT4 regulatory axis appears to be an important therapeutic target in OSCC.

\section{Results}

\subsection{Establishment of miR-31 Deletion Subclones}

The strategy used for deleting the miR-31 gene by means of the CRISPR/Cas9 system is shown in Figure S1A. Cleavage of Cas 9 at $5^{\prime}$ sgRNA and $3^{\prime}$ sgRNA cut sites should result in miR-31 gene truncation. SAS cells were selected with puromycin to obtain a total of 19 subclones lacking $m i R-31$ expression (Figure S1B). Sequencing of 10 subclones confirmed the deletion of nucleotides of various lengths between cut sites (Figure S1C). Deletion subclones were subjected to phenotypic analysis (Figure 1A). The results showed growth inhibition in the majority of subclones except for \#23, \#31, and \#70 (Figure 1B) and reduced invasion except for subclones \#31 and \#70 (Figure 1C). The colony formation capability was impaired in all tested subclones (Figure 1D). The expression of NUMB isoforms in deletion subclones were upregulated to varying extents. The increases in \#23, $\# 31$, \#34, \#55, and \#70 were modest (Figure 1E). Subclones \#13 and \#51 had slight decreases in the Numb1 and Numb 2 isoforms. The subcutaneous tumorigenicity of subclone \#55 in nude mice was significantly attenuated (Figure 1F). Thus, we concluded that the miR-31 gene locus was required to elicit oncogenesis in OSCC cells.

\subsection{Establishment of NUMB Deletion and Activation Systems}

The strategy used for deleting NUMB by means of the CRISPR/Cas9 system and a single sgRNA in OSCC cells is shown in Figure S2A. Sequencing of the established subclones of SAS and OECM1 confirmed the introduction of various Indel nucleotides which resulted in the frameshift and the occurrence of premature stop codons (Figure S2B). Western blot analysis revealed the nearly complete disappearance of NUMB protein expression in S1-S3 subclones established from SAS cells and O1-O3 subclones from OECM1 cells (Figure 2A). In general, these subclones exhibited increased competence in wound healing (Figure 2B) and colony formation (Figure 2C). 
A

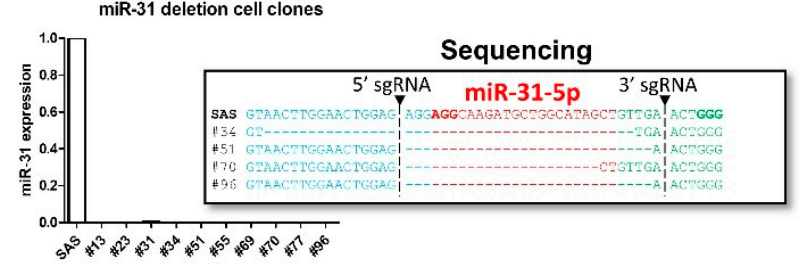

C
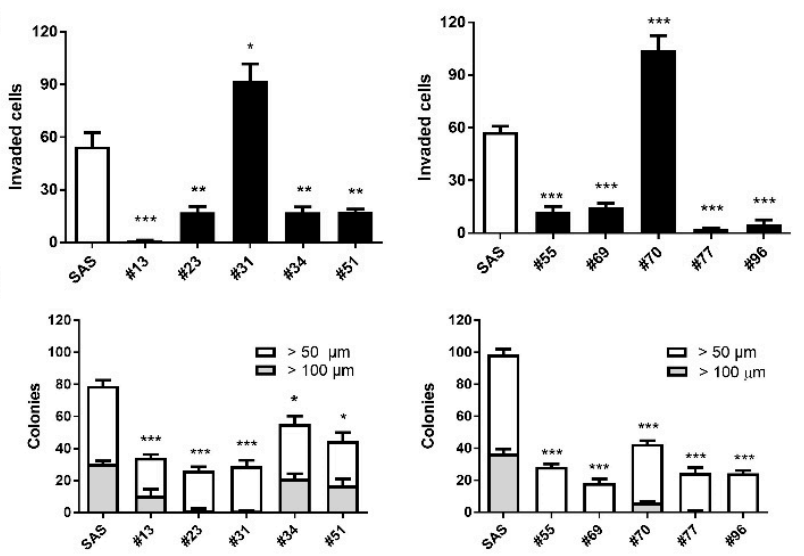

$\mathbf{E}$

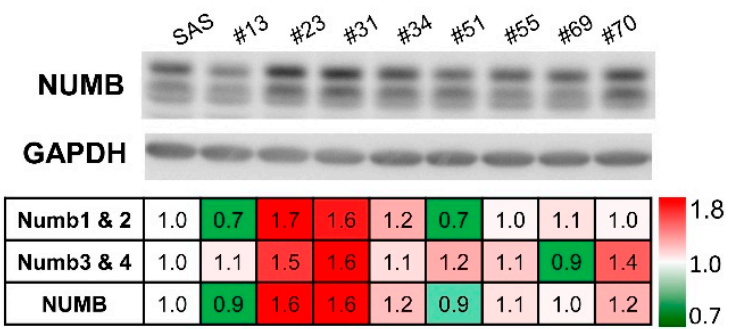

B

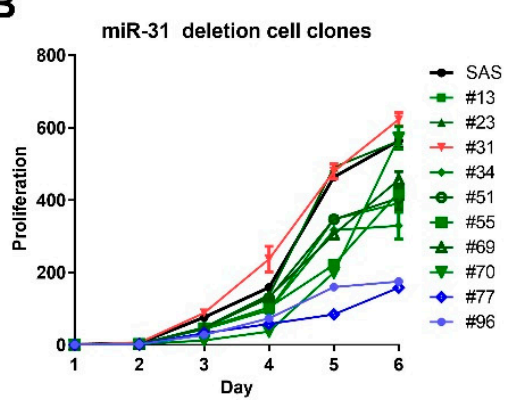

$\mathbf{F}$

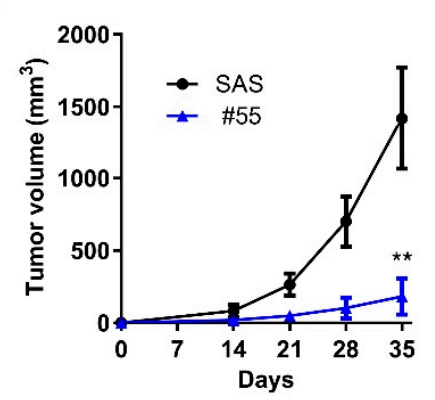

\begin{tabular}{|c|c|c|}
\hline \multirow{2}{*}{ SAS } & \multicolumn{2}{|c|}{ PD (h) } \\
\cline { 2 - 3 } & Time (h) & ratio \\
\hline Par & 18.5 & 1.0 \\
\hline \#13 & 23.8 & 1.3 \\
\hline \#23 & 14.4 & 0.8 \\
\hline \#31 & 19.7 & 1.1 \\
\hline \#34 & 25.0 & 1.4 \\
\hline \#51 & 29.0 & 1.6 \\
\hline \#55 & 22.6 & 1.2 \\
\hline \#69 & 26.9 & 1.5 \\
\hline \#70 & 13.0 & 0.7 \\
\hline \#77 & 33.4 & 1.8 \\
\hline \#96 & 38.4 & 2.1 \\
\hline
\end{tabular}

Fastest Control Slowest

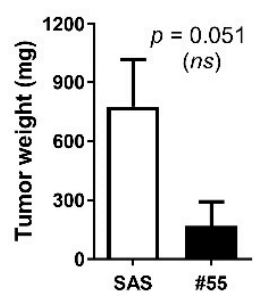

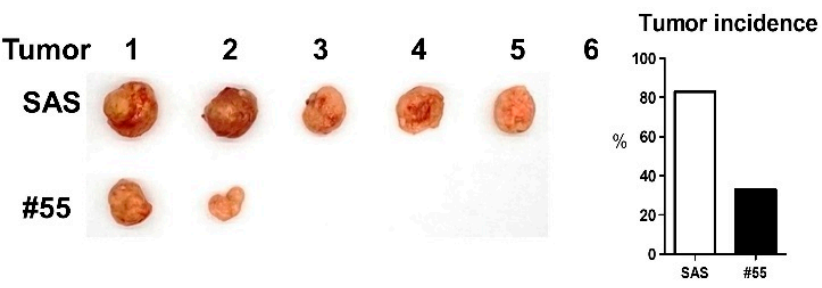

Figure 1. miR-31 deletion suppresses OSCC oncogenicity. (A) qRT-PCR analysis of $m i R-31$ expression in representative subclones revealed the complete absence of $m i R-31$ expression. Representative sequencing results, shown in the indent, demonstrated the truncation of miR-31-5p segments in the subclones. miR-31 expression levels and the truncation of miR-31-5p in other subclones are shown in Figure S1B,C. (B,C) Growth and invasion assays. The right panel of (B) is a heatmap illustrating the population doubling time of subclones. Growth or invasion was reduced in 8 of the 10 subclones. PD, population doubling time (h). (D) Anchorage-independent colony formation. Anchorage-independent growth was decreased in all subclones. (E) Upper panel, Western blot analysis of NUMB expression in subclones relative to parental cells. Lower panel, heatmap illustrating the expression levels. Upper bands, the signals of Numb1 and Numb 2 isoforms. Lower bands, the signals of Numb3 and Numb4 isoforms. NUMB signals were increased in the analyzed subclones except for \#13 and \#51. (F) Tumorigenicity of subclone \#55. Upper left panel, the growth curve measured weekly. Upper right panel, the tumor weight at week 5 . Lower left panel, the images of harvested tumors. Lower right panel, the incidence of tumor induction. $n s$, not significant; ${ }^{*}, p<0.05 ;{ }^{* *}, p<0.01 ;{ }^{* * *}, p<0.001$.

A synergistic activation mediator (SAM) activation strategy was adopted to increase endogenous NUMB expression by promoter transactivation in SAS cells (Figure S3). Among the eight potential sites used to test SAM activity, robust upregulation of NUMB mRNA and protein expression was achieved by dCas9-SAM\#6 in SAS cells but not in unstimulated parental cells (Figure 2D). The upregulation of endogenous NUMB expression was significantly associated with decreased wound healing, invasion, and colony formation of SAS cells (Figure 2E). 
A

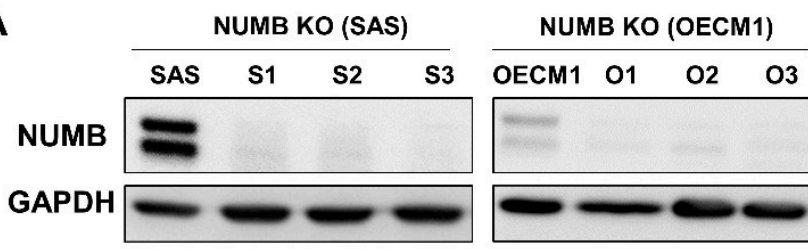

B
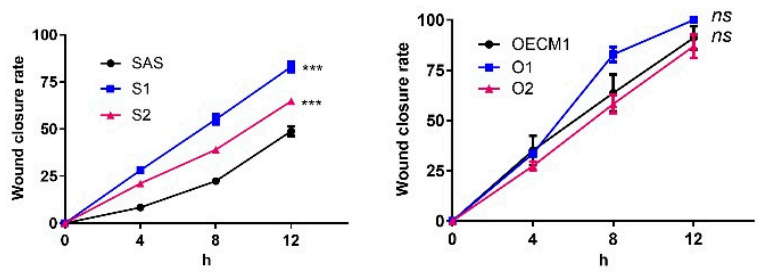

C
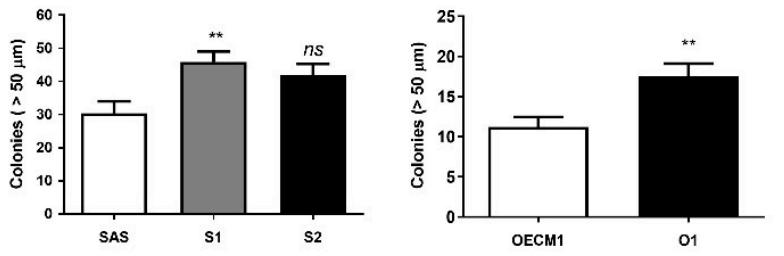

D

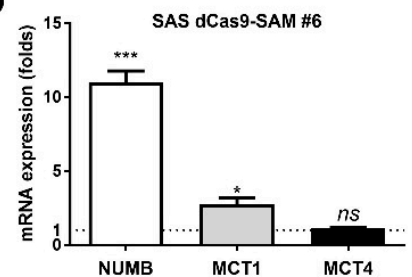

E
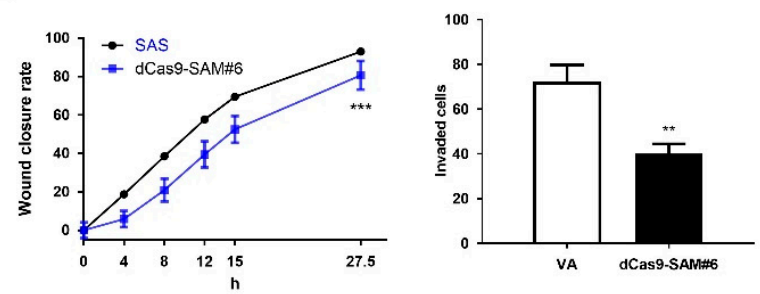

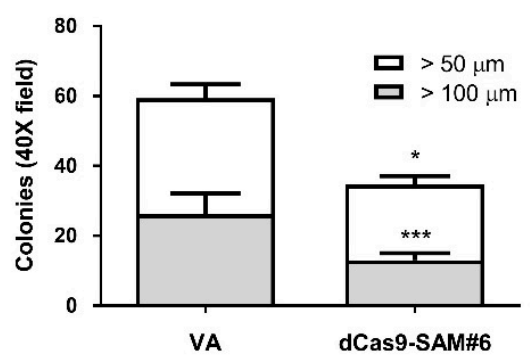

Figure 2. NUMB deletion increases the OSCC oncogenicity. (A) Western blot analysis to detect the near absence of NUMB signals in the S1-S3 subclones of SAS cells and O1-O3 subclones of OECM1 cells compared to parental cells. (B) Wound healing assay. There was a conspicuous increase in wound closure in the $\mathrm{S} 1$ and $\mathrm{S} 2$ subclones but no changes in the $\mathrm{O} 1$ and $\mathrm{O} 2$ subclones. (C) Anchorage-independent colony formation. It shows the increased colony formation in S1, S2, and O1 subclones relative to controls. (D) The induction of endogenous NUMB expression using the NUMB dCas9-SAM system in SAS cells. The delivery of sgRNA\#6 to transactivate the NUMB promoter increased NUMB mRNA (left panel) and protein (right panel) expression. (E) Elevated NUMB expression from the endogenous locus decreased the wound closure rate (upper left panel), invasion (upper right panel), and colony formation (lower panel) of SAS cells. VA, vector alone. ns, not significant;,$p<0.05 ; * *, p<0.01 ; * * *, p<0.001$.

\subsection{Numb1 or Numb4 Expression Represses the Colony Formation of NUMB Deletion Subclones}

The structures of Numb1-Numb4 isoforms frequently present in cells are illustrated in Figure 3A. Compared to Numb1, which is the complete NUMB isoform, the Numb4 isoform lacks 59 residues in functional domains PTB and PRR and its molecular weight is approximately $7 \mathrm{kDa}$ smaller. Parental SAS and OECM1 cells and S1 and O1 subclones were transfected with Numb1 and Numb4 isoform constructs which we previously established [23]. Western blot analysis revealed the differential expression of these proteins in cells (Figure 3B). Colony formation assays indicated that either Numb1 or Numb4 expression repressed colony formation in SAS or OECM1 cells (Figure 3C-E). The increased colony formation phenotype of $N U M B$-deleted subclones $\mathrm{S} 1$ and $\mathrm{O} 1$ was also reversed by the expression of either Numb1 or Numb4. Altogether, we concluded that Numb1 and Numb4 isoforms resulted in comparable reductions in colony formation in OSCC cells. 
A

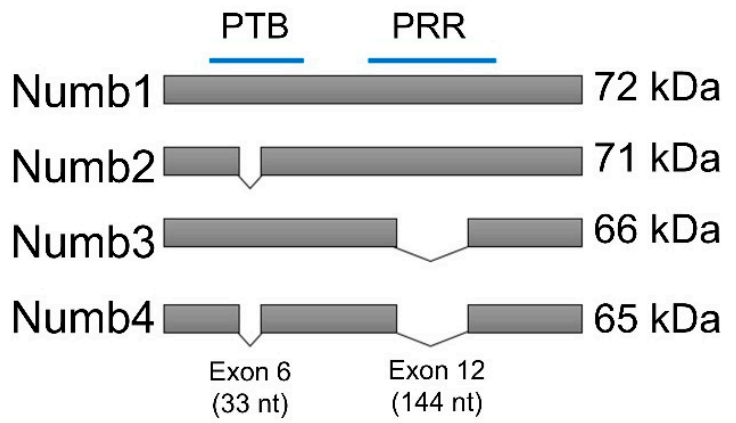

C
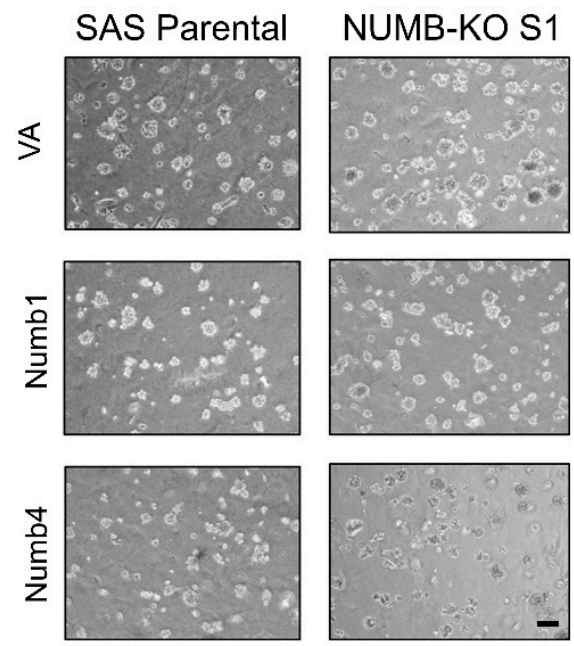

D

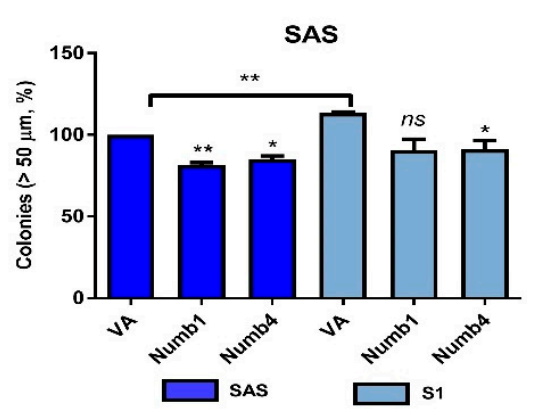

$\mathbf{E}$

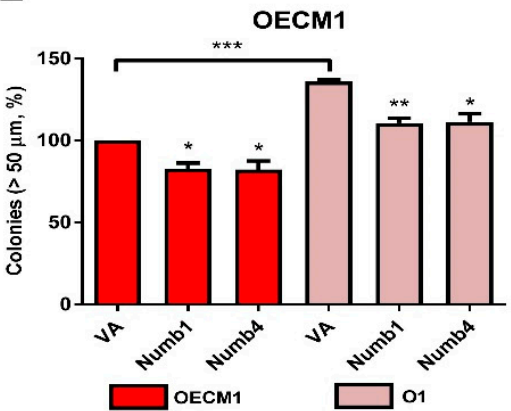

Figure 3. Exogenous expression of Numb1 and Numb4 isoforms increases colony formation in NUMB-deleted OSCC cells. (A) Schematic diagram illustrating the differences between NUMB isoforms. (B) Western blot analysis. This indicates differential expression of exogenous Numb1 or Numb4 in SAS, S1, OECM1, and O1 cells. (C) Representative photomicrographs of anchorage-independent colonies of SAS cells following NUMB knockout or the exogenous expression of Numb1 or Numb4. Bar, $200 \mu \mathrm{M}$. (D) Quantitation of colonies in SAS cells (left) and (E) OECM1 cells (right). Percentage grading was used to integrate three or four individual assays. VA, vector alone. $n s$, not significant; ${ }^{*}, p<0.05 ;{ }^{* *}, p<0.01{ }^{* * *}, p<0.001$.

\subsection{Reduced NUMB Expression Upregulates MCT1/MCT4 Expression and Glycolytic Respiration}

Western blot analysis indicated the upregulation of MCT1 in S1, S3, O1, and O2 subclones relative to parental cells and the upregulation of MCT4 in S2, S3, O1, O2, and O3 subclones relative to parental cells (Figure 4A). However, GLUT1 and GLUT3 expression in OSCC cells was not changed following the deletion of NUMB. Lactate production (Figure 4B) and anaerobic respiration (Figure $4 \mathrm{C}$ ) increased in $N U M B$-deleted subclones. To confirm the effects of $N U M B$, knockdown of $N U M B$ was carried out in OSCC cells. We found that MCT1/MCT4 expression (Figure 4D) and lactate production (Figure 4E) increased when $N U M B$ was knocked down in SAS and OECM1 cells. 


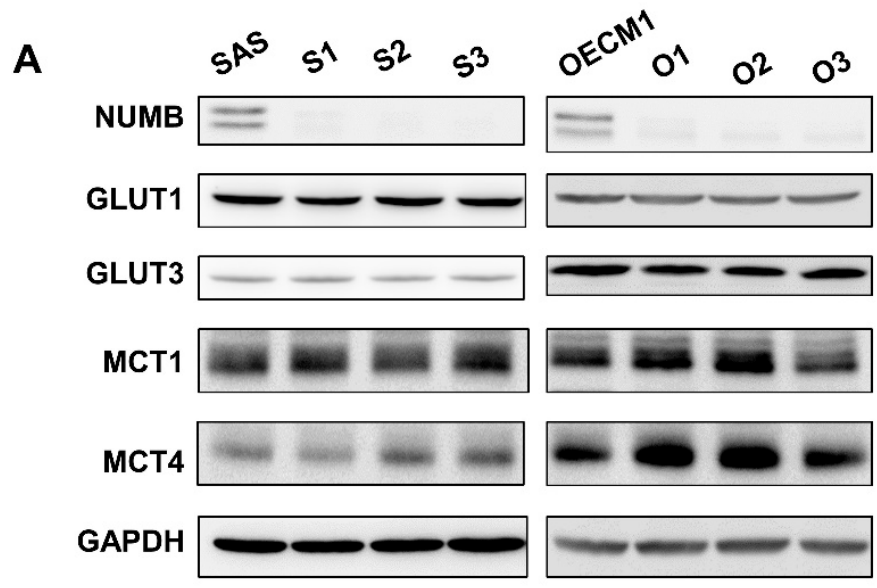

B
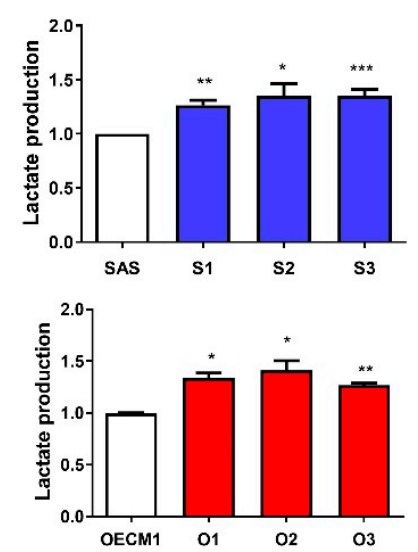

C
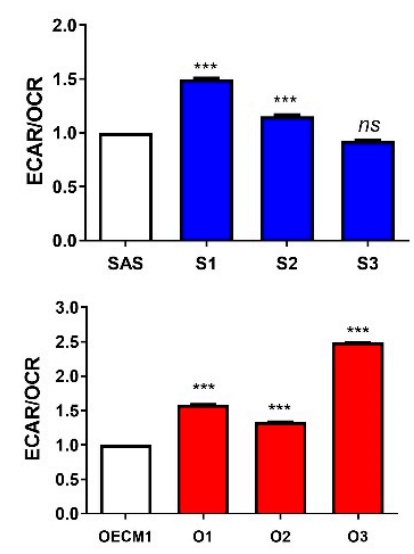

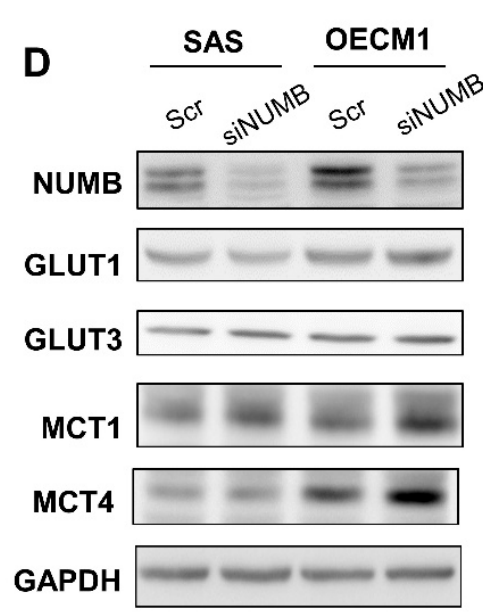

E

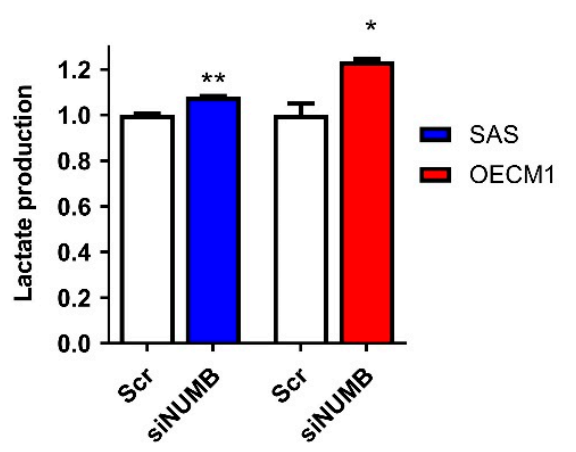

Figure 4. NUMB deletion or knockdown increases the expression of MCT1/MCT4 and glycolysis in OSCC cells. (A,D) Western blot analysis of knockout subclones and knockdown cells. There was a general tendency towards increased MCT1 and MCT4 expression in NUMB-deleted or NUMB-knockdown SAS and OECM1 cells. The expression of GLUT1 and GLUT3 was not changed. (B,E) Lactate production assay of knockout subclones and knockdown cells. The NUMB-deleted subclones and knockdown cells exhibited increased lactate production. (C) The anaerobic respiration shown by the ECAR/OCR ratio, which was measured by Seahorse flux analysis, increased in most NUMB knockout subclones. Scr, scramble oligonucleotide. $n s$, not significant; ${ }^{*}, p<0.05 ;{ }^{* *}, p<0.01 ;{ }^{* * *}, p<0.001$.

\subsection{MCT1 and MCT4 Increase the Oncogenicity of OSCC Cells}

Exogenous expression and knockdown of MCT1/MCT4 were carried out in SAS and OECM1 cells. Western blot analysis confirmed exogenous expression and knockdown in SAS cells (Figure 5A). In SAS cells, exogenous MCT1 expression increased migration (Figure 5B), while exogenous MCT4 expression increased invasion (Figure 5C). Both MCT1 and MCT4 increased colony formation when expressed exogenously (Figure 5D). The knockdown of MCT1 or MCT4 expression drastically decreased colony formation (Figure 5E). In OECM1 cells, knockdown of MCT1 or MCT4 expression markedly decreased growth and invasion (Figure 5F,G). Exogenous MCT1 or MCT4 expression increased the invasion of OECM1 cells (Figure 5H). In summary, the results suggest that MCT1 and MCT4 enhance oncogenicity in OSCC cells. In the GSE37991 Gene Expression Omnibus (GEO) database, upregulation of both MCT1 and MCT4 was noted in OSCC tumors (Figure S4A). Although the correlation between the expression of MCT1 and MCT4 is lacking, the vast majority ( $75 \%$ ) of tumors upregulated MCT1 and MCT4 simultaneously. In The Cancer Genome Atlas (TCGA) HNSCC dataset, a significant correlation between the expression of both MCT1 and MCT4 was found (Figure S4B). In addition, higher MCT1 or MCT4 expression in tumors was associated with the worse survival of patients. Tu- 
mors exhibiting high expression of both $M C T 1$ and MCT4 displayed even worse patient survival. Thus, oncogenic roles of MCT1 and MCT4 are supported by the findings in patient-derived tissues.

A

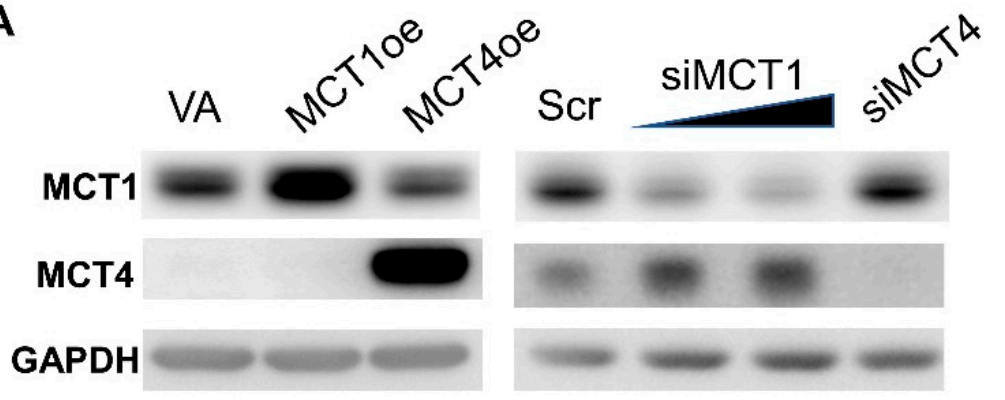

B

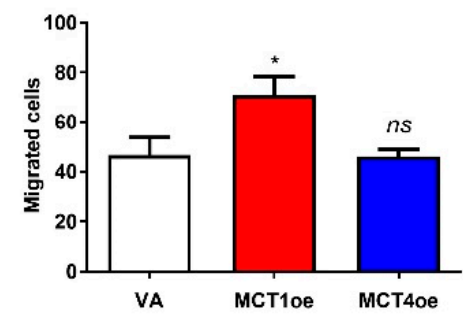

C

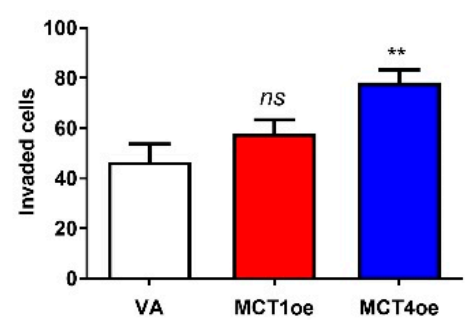

D

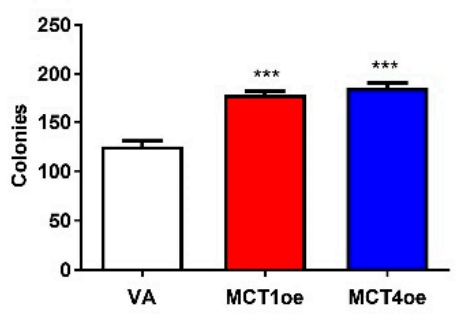

$\mathbf{E}$

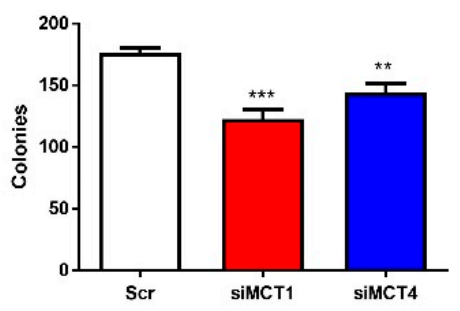

$\mathbf{F}$
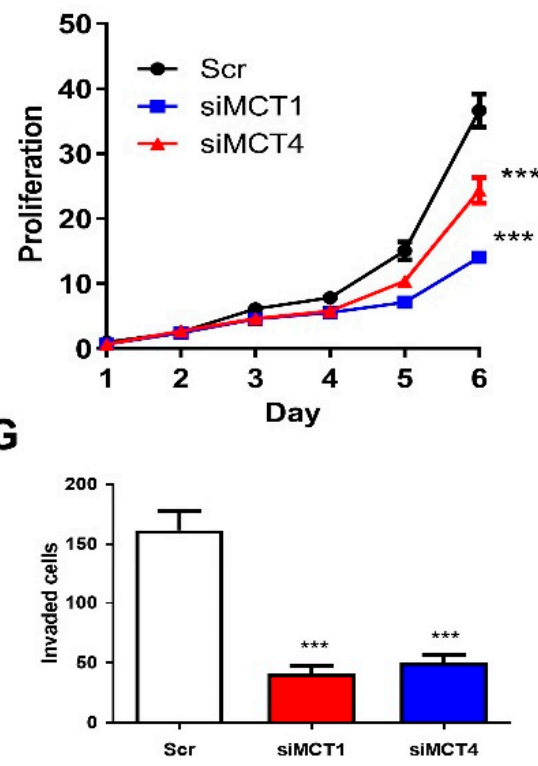

H

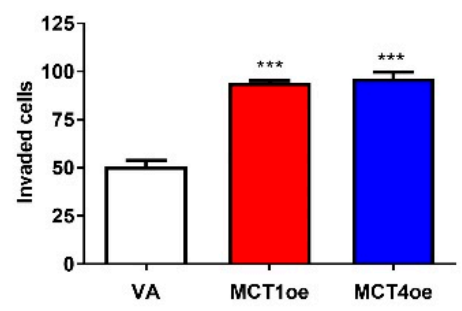

Figure 5. Association between MCT1/MCT4 expression and OSCC oncogenicity. (A-E) SAS cells. (F-H) OECM1 cells. (A) Western blot analysis to confirm the exogenous expression or knockdown of MCT1 or MCT4 in SAS cells. The signal intensity of MCT4 image in left panel was attenuated to acquire better illustration. Regulation between MCT1 and MCT4 seemed to exist. (B) Migration assay. (C,G,H) Invasion assay. (D,E) Anchorage-independent colony formation. (F) Proliferation. (B-D) Exogenous MCT1 or MCT4 expression generally increased the migration, invasion and colony formation of SAS cells. (E) Knockdown of MCT1 or MCT4 significantly decreased colony formation in SAS cells. (F) Knockdown of MCT1 or MCT4 decreased the proliferation of OECM1 cells. (G,H) Knockdown of MCT1 or MCT4 decreased invasion, while exogenous expression increased the invasion of OECM1 cells. Scr, scramble oligonucleotide; VA, vector alone. ns, not significant; ${ }^{*}, p<0.05 ; * *, p<0.01 ; * * *, p<0.001$.

\subsection{NUMB4 Binds MCT1/MCT4 and Induces Polyubiquitination}

Treatment of 293T cells with MG132 increased the abundance of HIF1 $\alpha$ and NUMB, probably due to the inhibition of proteasomal activity (Figure 6A). Numb4, MCT1, or MCT4 were exogenously expressed beyond the endogenous levels when appropriate plasmids were transfected either by themselves or in combination. The net changes in MCT1 and MCT4 following treatment are difficult to evaluate since HIF1 $\alpha, \mathrm{NUMB}$, and MG132 can regulate MCT1/MCT4 expression [36]. In 293T cells co-expressing Numb4 and MCT1, the analysis of immunoprecipitates isolated by anti-NUMB antibody or antiMCT1 antibody detected signals of MCT1 and NUMB, respectively (Figure 6B, upper panels). In cells co-expressing Numb4 and MCT4, the analysis of immunoprecipitates 
isolated by anti-NUMB antibody or anti-MCT4 antibody detected signals of MCT4 and NUMB, respectively (Figure 6B, lower panels). The results indicate that Numb4 can bind MCT1 and MCT4. In the immunoprecipitates isolated by anti-MCT1 antibody in SAS cells expressing ubiquitin and Numb4, polyubiquitination of MCT1, and co-precipitated NUMB increased (Figure 6C). In the immunoprecipitates isolated by anti-MCT4 antibody in 293T cells expressing ubiquitin and Numb4, polyubiquitination of MCT4, and NUMB increased and MCT4 decreased (Figure 6D). These observations indicate that Numb4 is involved in the ubiquitination of MCT1 and MCT4.

A

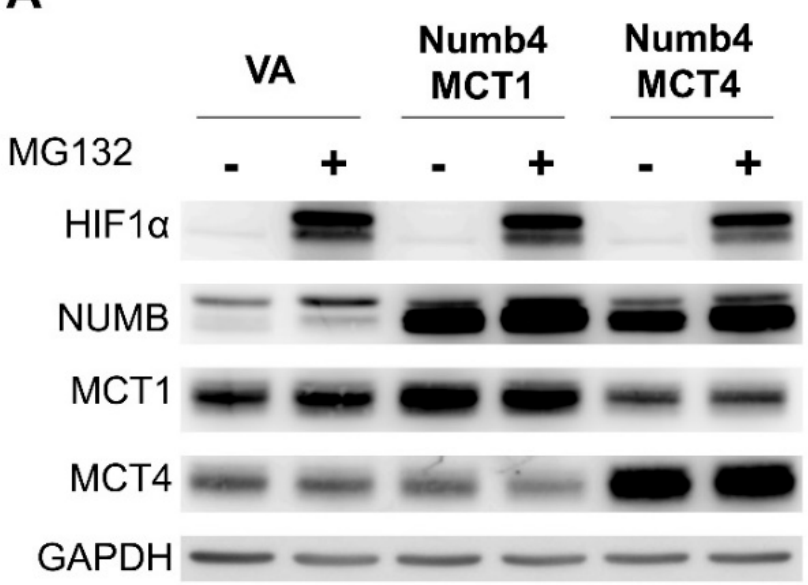

C

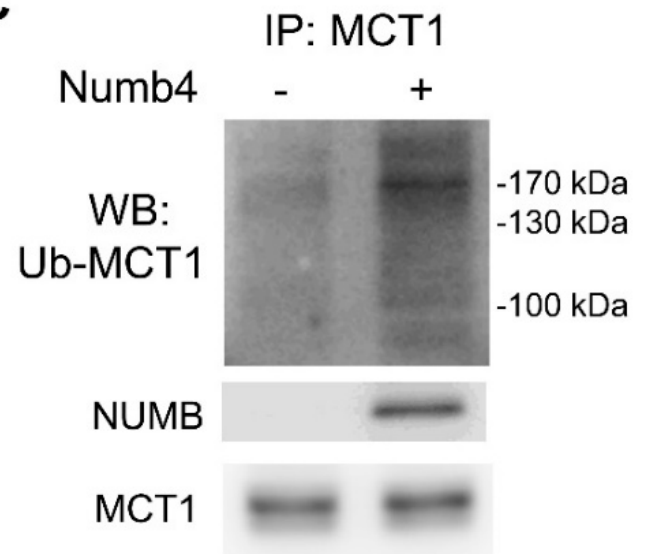

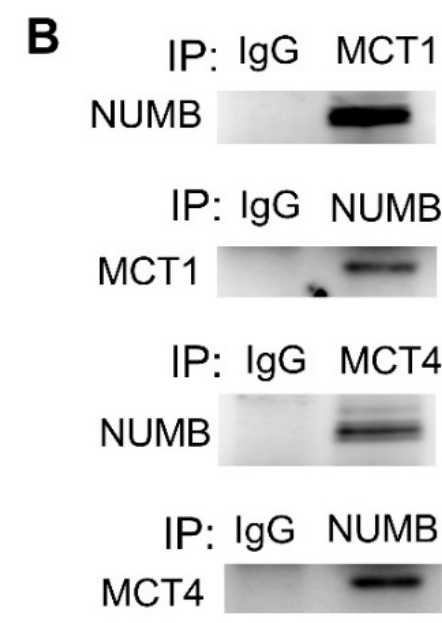

D

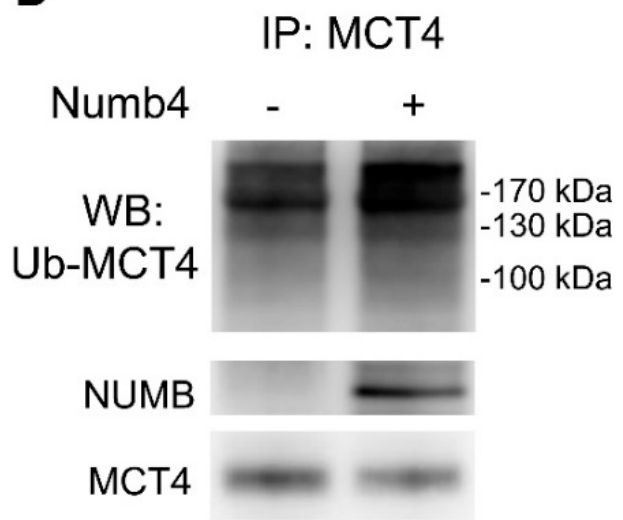

Figure 6. Numb4 binds MCT1 and MCT4 and facilitates their polyubiquitination. (A) Western blot analysis of 293T cells transfected with Numb4, MCT1, and MCT4 constructs and treated with MG132. HIF1 $\alpha$ expression serves as a control to validate the efficacy of MG132. Treatment with $20 \mu \mathrm{M}$ MG132 for $8 \mathrm{~h}$ increased the abundance of endogenous and exogenous NUMB, but it did not consistently affect exogenous or endogenous MCT1 or MCT4. Exogenous MCT4 expression decreased the abundance of endogenous MCT1. (B) Immunoprecipitation. Immunoprecipitates generated by anti-NUMB antibody contained MCT1 and MCT4 in 293T cells. Immunoprecipitates generated by anti-MCT1 or anti-MCT4 antibody also contained NUMB. (C) Enhanced polyubiquitination of MCT1 in the immunoprecipitates generated by anti-MCT1 following Numb4 expression in SAS cells. (D) Enhanced polyubiquitination of MCT4 in the immunoprecipitates generated by anti-MCT4 following Numb4 expression in 293T cells. VA, vector alone.

\section{7. miR-31- and NUMB-Deletion Phenotypes Are Reversed by Modulating MCT1/MCT4 Expression}

To address the effects of the miR-31-NUMB-MCT1/4 axis in regulating tumor phenotypes and lactate production, the miR-31-deleted KO\#51 subclone, NUMB-deleted S1 subclone, and parental SAS cells were studied. In KO\#51 cells, the reduced migration, invasion, colony formation, and lactate production were rescued by MCT1 or MCT4 expression 
to varying extents (Figure 7A-D). The effects of MCT4 expression on oncogenicity were particularly prominent. Likewise, the phenotypes and lactate production capability of the S1 subclone were reversed by the knockdown of MCT1 or MCT4 (Figure 7E-H). The effects of MCT4 on invasion and colony formation were particularly prominent. These functional clues substantiate the existence of the miR-31-NUMB-MCT1/4 regulatory axis in OSCC.

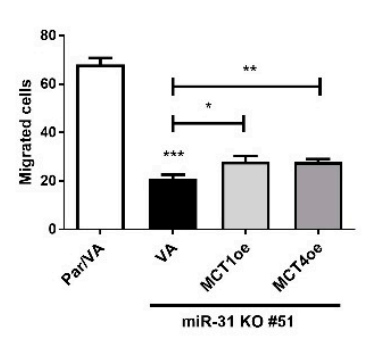

E

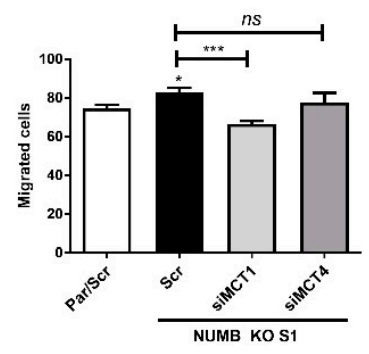

A

B

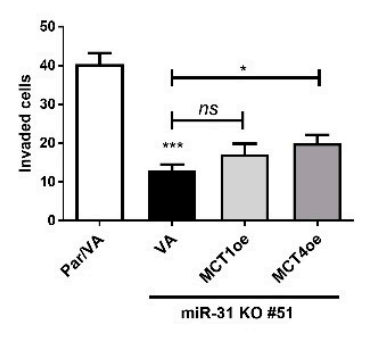

F

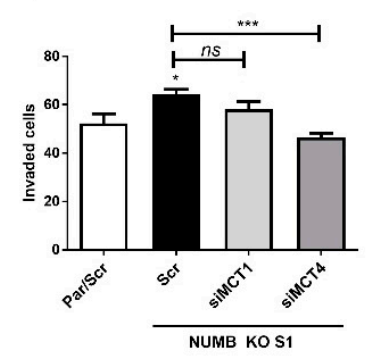

C

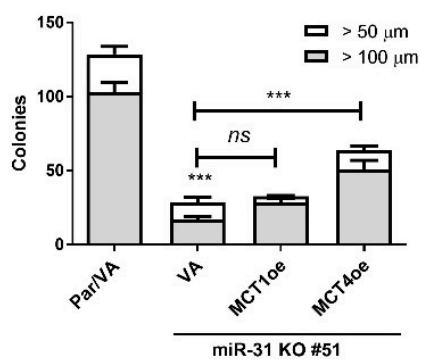

G

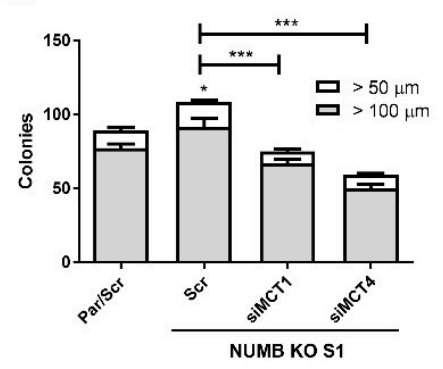

D

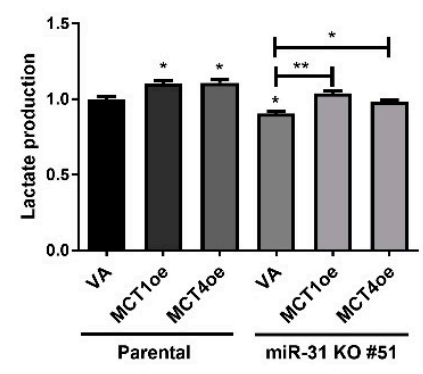

H

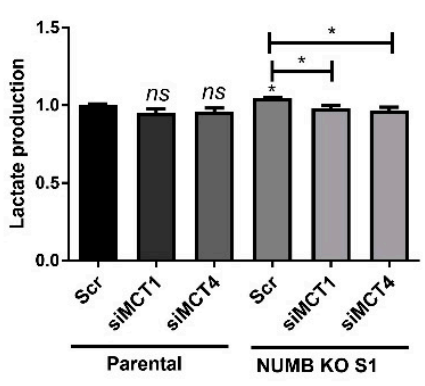

Figure 7. Reversal of miR-31-NUMB-associated tumor phenotypes and lactate production by MCT1 and MCT4. (A-D) SAS cell and SAS miR-31 KO\#51 subclone. (E-H) SAS cells and SAS NUMB KO S1 subclone. (A,E) Migration assay. (B,F) Invasion assay. (C,G) Anchorage-independent colony formation. (D,H). Lactate production. The decreased oncogenicity and lactate production due to miR-31 deletion was partially reversed by exogenous expression of MCT1 or MCT4. The increased oncogenicity and lactate production due to NUMB deletion was partially reversed by the knockdown of MCT1 or MCT4 expression. Scr, scramble oligonucleotide; VA, vector alone. $n s$, not significant; ${ }^{*}, p<0.05 ;{ }^{* *}, p<0.01 ;{ }^{* *}, p<0.001$.

\section{Discussion}

A novel finding in the present work is that NUMB affected lactate production and glycolytic respiration in OSCC cells. In previous studies, miR-31 was shown to target SIRT3 to inhibit mitochondrial activity [7] and target $A C O X 1$ to disrupt the lipidome profile in OSCC [8]. Other studies reported that miR-31 modulated the metabolic profile by targeting different types of cells $[10,11,37]$. As $N U M B$ is the direct target of $m i R-31$ and several other oncogenic miRNAs $[23,25,26]$, findings in this work substantiated that $m i R-31$ is an oncogenic miRNA that also modulates complex metabolic regulation in OSCC $[7,8]$. Inhibition of $N U M B$ by miR-31 also exerts both oncogenic and metabolic effects. We validated the efficacy of miR-31 deletion using a double sgRNA-guided CRISPR/Cas9 cleavage approach [38]. This strategy can be further improved to achieve efficient miR-31 blockade for in vivo tumor therapy.

Although NUMB has been known to affect complex controls on cellular functions, including oncogenesis and mitochondria [22,39], the current study employed NUMB deletion to gain insight into the function of NUMB in OSCC cells. The increased lactate production and the glycolytic metabolic switch appeared to coincide with the increase in MCT1/MCT4 protein levels in NUMB-deleted subclones. In line with these findings, the knockdown of $N U M B$ and induction of endogenous $N U M B$ expression also yielded results suggesting that NUMB regulated MCT1/MCT4 expression and lactate production. Since the knockdown of 
MCT1/MCT4 also rescued the phenotypic and metabolic changes in NUMB-deleted cells, MCT1/MCT4 may modulate metabolic reprogramming and oncogenesis in addition to lactate flux $[32,33,35]$. The dual inhibition of MCT1/MCT4 ameliorated tumor growth [33]. In this study, we showed that NUMB concomitantly inhibited MCT1 and MCT4, which would inhibit neoplastic growth.

NUMB acts as an adaptor to promote polyubiquitination and induce degradation of various essential factors [14,16,19-22]. We identified the binding between Numb4 and MCT1/MCT4 in 293T cells and the increased polyubiquitination of MCT1/MCT4. Furthermore, MCT1/MCT4 protein abundance increased when NUMB was silenced in cells. Thus, the increase in lactate production following $N U M B$ deletion is likely due to the increase in MCT1/MCT4 $[29,30]$. However, the role of NUMB on the mitochondrial machinery underlying the respiratory switch awaits further investigation. CD44, CD147, or $\beta$-integrin interact with MCTs to act as chaperones or functional patterners [40-42]. Although the entire NUMB protein was required for the binding of NUMB with mGluR5 [28], during transdifferentiation from mesodermal cells to endothelial progenitor cells, the splicing factor mediated the induction of $N U M B \_S$ isoforms to regulate Notch signaling [27]. The present study demonstrated, for the first time, the presence of Numb4 and MCT1/MCT4 complexes, which may trigger proteasome degradation, although the PTB and PRR domains were absent in Numb4 [12]. As NUMB does not possess ubiquitin ligase activity [18], further studies are required to elucidate the interaction sites in the NUMB protein and the mechanisms of ubiquitin ligase recruitment for degradation.

Although the different NUMB isoforms may be responsible for diverse cellular phenotypes [27], our previous studies demonstrated that isoforms Numb1 and Numb4 possessed comparable suppressor activity in OSCC cells expressing endogenous NUMB [23]. The present study further established that both Numb1 and Numb4 were equally suppressive in OSCC subclones whose NUMB genome had been deleted. The knockout model can be valuable to specifically evaluate the activity of each individual isoform in the disease process [27]. Our NUMB knockout approach adopted a solitary sgRNA-guided DNA cleavage and recombination. However, diverse and complex Indel patterns were induced around the cleavage site in the $N U M B$ genome in subclones. This could be the reason why the faint NUMB signals were observed. Alternatively, a small fraction of functionally silent parental cells may be still present in the subclones. A faint signal may also be an artifact caused by the cross-reactivity of the antibody. As the suppressor role of NUMB is definitive, endogenous NUMB induction using the CRISPR/dCas-SAM system being tested in this study would be a potential option for cancer therapy.

Lactate is a crucial fuel source required for sustained energy support in tumor cells [32]. In addition to the modulation of lactate flux, the vital roles of MCT1/MCT4 in OSCC tumorigenesis were defined in this study. In agreement with a previous study [35], our database analysis also indicated the concurrent high expression of MCT1 and MCT4 in HNSCC, which defined poor patient prognosis. There were at least 70 genes whose aberrant expression highly correlated with MCT1 expression in the TCGA HNSCC tumor cohort [33]. Although it is still not clear whether MCT-mediated oncogenic or metabolic shifts are due to the direct regulation of downstream effectors, they may interact with each other and also with partners, and disrupt metabolic homeostasis related to lactate flux [40-42]. Therefore, the inhibition of MCTs could be a promising therapeutic option $[33,36]$.

\section{Material and Methods}

\subsection{Cell Lines and Reagents}

SAS and OECM1 OSCC cell lines along with 293T cells were cultured as previously described $[1,2,25,43]$. Small interfering RNA oligonucleotides (Table S1) and their scramble (Scr) controls were purchased from Thermo Fisher Scientific (Waltham, MA, USA). The doses of oligonucleotides have been validated as 60 or $120 \mathrm{nM}$. TransFectin ${ }^{T M}$ Reagent (BioRad, Hercules, CA, USA) was used for transfection. Unless specified, all reagents were obtained from Sigma-Aldrich (St. Louis, MO, USA). 


\section{2. $q R T-P C R$ Analysis}

TRI reagent (Molecular Research Center, Cincinnati, OH, USA) was used to isolate RNA from cells. TaqMan miRNA assay kits (Apply Biosystems, Waltham, MA, USA) were used to quantify the expression of miR-31, NUMB, MCT1, and MCT4 using RNU6B or GAPDH as internal controls (Table S2). The difference in gene expression between samples was calculated using the $2^{-\Delta \Delta C t}$ method, where $\mathrm{Ct}$ is the threshold cycle [2].

\subsection{Western Blot}

Cell lysates were subjected to Western blot analysis using appropriate primary and secondary antibodies (Table S3). Signals of tested proteins were normalized to GAPDH to quantify expression levels [2].

\subsection{Clustered Regularly Interspaced Short Palindromic Repeat (CRISPR)/Cas9 Gene Edition}

The oligonucleotides were annealed to form double-stranded $5^{\prime}$ and $3^{\prime} \operatorname{sgRNAs}$ to delete mature hsa-miR-31-5p (Table S4). Each sgRNA was cloned into the pU6-sgRNA.pPuro vector (National RNAi Consortium, Taipei, Taiwan) and co-transfected with the p5wCas9.pBsd vector (National RNAi Consortium) into cells (Figure S1A). After puromycin selection, single cells isolated by limiting dilution were expanded to obtain subclones [38]. qRT-PCR was used to detect miR-31 expression. PCR products encompassing the deletion segment in cell subclones (Table S5) were cloned into the pHE vector (Addgene, Cambridge, MA, USA), and plasmid DNA from multiple bacterial colonies was sequenced to ascertain the presence of $m i R-31$ deletion in cell subclones [43].

The pAll-NUMB-Cas9-Ppuro vector, which co-expresses Cas9 and sgRNA targeting NUMB (Table S4), was obtained from the National RNAi Consortium (Figure S2A). A pSurrogate reporter vector (National RNAi Consortium) was used to confirm the effectiveness of the CRISPR/Cas9 system [43]. After selection with puromycin, cells expressing red fluorescence from mCherry were sorted and expanded to establish subclones. In addition, PCR products encompassing the deletion segment in cell subclones were cloned into a bacterial vector (Table S5), and plasmid DNA from multiple bacterial colonies was sequenced to ascertain the presence of $N U M B$ deletion in cell subclones [43].

\subsection{Induction of NUMB Activation Using Crispr-dCas9 SAM System}

The potential sequence segments allowing $\mathrm{dCas} 9$ recognition within $\sim 250$ bp upstream of the NUMB transcription start site were predicted by the E-crisp (http:/ / www.e-crisp. org /E-CRISP / assessed date: 20 September 2017) module [44]. Eight oligonucleotides containing sgRNAs that recognize these sequences were ligated into the sgRNA (MS2) cloning backbone (Addgene) to generate $S A M$ constructs for promoter activation (Figure S3). The dCas9-SAM\#6 construct was validated as the most potent construct for NUMB induction in pilot tests (Table S4).

\subsection{Plasmid Construction and Overexpression}

The coding sequences of MCT1, MCT4, and ubiquitin transcripts were amplified by PCR and cloned into pcDNA3.1(+) or pcDNA3.1(-) vectors for exogenous expression (Table S5). The vectors used for the overexpression of Numb1 and Numb4 variants were previously established by our group [23].

\subsection{Phenotypic and Tumorigenic Assays}

Cell proliferation, wound closure, migration, invasion, and anchorage-independent colony formation experiments were carried out according to previously published protocols [23]. For migration and invasion assays using a Transwell apparatus, cell growth was arrested by treatment with $1 \mu \mathrm{M}$ hydroxyurea. Unless specified, migrated cells and invaded cells in 400X image fields and the colonies in 100X image fields were counted. For the induction of subcutaneous xenografts, $5 \times 10^{5}$ cells were injected into the flanks of nude mice. Tumor volumes were calculated using the formula: volume $=0.5 a b^{2} ; a$, the 
longest diameter, $b$, the shortest diameter [2,43]. This animal study was approved by the Institutional Animal Care and Use Committee (IACUC) of National Yang-Ming University (IACUC approval no.: 1070503).

\subsection{Co-Immunoprecipitation (Co-IP)}

Anti-NUMB, anti-MCT1, or anti-MCT4 antibodies were conjugated with protein A magnetic beads according to the protocol provided by the supplier (Pierce ${ }^{\mathrm{TM}} \mathrm{Classic}$ Magnetic IP/Co-IP Kit, Thermo Fisher Scientific). Mouse or rabbit IgG were used as negative controls (Table S6). After transfection with overexpression plasmids, $500 \mu \mathrm{g}$ of cell lysate isolated from SAS or 293T cells were used as input for immunoprecipitation. After incubating input with pre-coupled beads, proteins on the beads were extracted with a sample buffer. All samples were boiled for $5 \mathrm{~min}$ before Western blotting analysis.

\subsection{Measurement of Lactate Production and Mitochondrial Respiration}

$\mathrm{L}(+)$-lactate in culture medium obtained from equal numbers of cells was measured by a lactate colorimetric assay kit (BioVision, Milpitas, CA, USA). The mitochondrial respiration of cells was measured by a Seahorse XF24 Extracellular Flux system following the protocols provided by the supplier (Seahorse Bioscience, North Billerica, MA, USA). The oxygen consumption rate (OCR) and extracellular acidification rate (ECAR) were determined by a calculation software provided with the appliance [7].

\subsection{Statistical Analysis}

Data are shown as the mean \pm SE. The Mann-Whitney test, $t$-test, and two-way ANOVA were performed. The GEO database was assessed through the website (http: / /www.ncbi.nlm.nih.gov/geo/assessed date: 28 February 2019). The genes in the HNSCC subset of the TCGA database were analyzed using UCSC Xena Functional Genomics Explorer (https: / / xenabrowser.net/assessed date: 24 July 2018), linear correlation analysis and Kaplan-Meier survival curves. $p<0.05$ was considered statistically significant.

\section{Conclusions}

In conclusion, this study identified the presence of the miR-31-NUMB-MCT1/MCT4 axis in mediating oncogenesis and metabolic switching, which implies that disruption of this cascade may intercept tumor pathogenesis and aerobic glycolysis.

Supplementary Materials: The following are available online at https://www.mdpi.com/article/10 .3390/ijms222111731/s1.

Author Contributions: Conceptualization, C.-H.C., S.-C.L. and K.-W.C.; methodology, C.-H.C., C.Y.F.C.; software, C.-C.Y., Y.-C.L., S.-R.C.; formal analysis, C.-H.C., C.-Y.F.C.; investigation, C.-H.C., C.-Y.F.C.,Y.-C.L., S.-R.C.; resources, S.-C.L. and K.-W.C., C.-C.Y.; writing-original draft preparation, C.-H.C., S.-C.L. and K.-W.C.; writing-review and editing, C.-H.C., S.-C.L. and K.-W.C.; funding acquisition, C.-H.C., S.-C.L. and K.-W.C. All authors have read and agreed to the published version of the manuscript.

Funding: This study was supported by grants MOST107-2314-B-010-026-MY3 and MOST107-2314B-010-032-MY3 and postdoctoral supporting grant MOST109-2811-B-010-534 for Dr. C.-H.C. from Ministry of Science and Technology, Taiwan.

Acknowledgments: We acknowledge the helps from Wan-Chun Li and Yi-Ta Hsieh.

Conflicts of Interest: The authors declare no conflict of interest. 


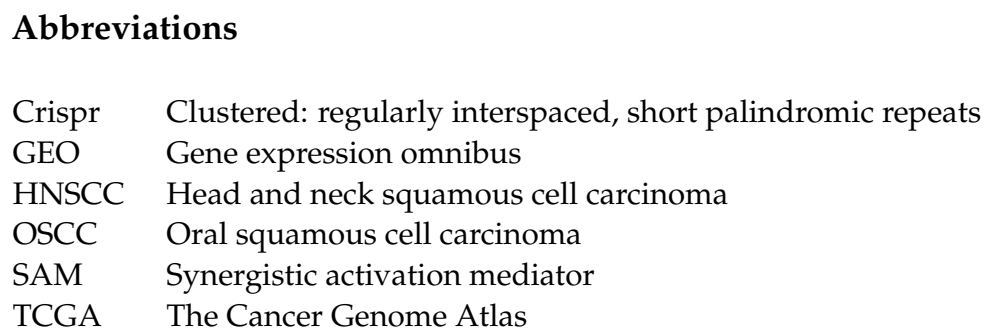

\section{References}

1. Chen, Y.F.; Yang, C.C.; Kao, S.Y.; Liu, C.J.; Lin, S.C.; Chang, K.W. MicroRNA-211 enhances the oncogenicity of carcinogen-induced oral carcinoma by repressing TCF12 and increasing antioxidant activity. Cancer Res. 2016, 76, 4872-4886. [CrossRef] [PubMed]

2. Liu, C.J.; Tsai, M.M.; Hung, P.S.; Kao, S.Y.; Liu, T.Y.; Wu, K.J.; Chiou, S.H.; Lin, S.C.; Chang, K.W. miR-31 ablates expression of the HIF regulatory factor FIH to activate the HIF pathway in head and neck carcinoma. Cancer Res. 2010, 70, 1635-1644. [CrossRef]

3. Tseng, S.H.; Yang, C.C.; Yu, E.H.; Chang, C.; Lee, Y.S.; Liu, C.J.; Chang, K.W.; Lin, S.C. K14-EGFP-miR-31 transgenic mice have high susceptibility to chemical-induced squamous cell tumorigenesis that is associating with Ku80 repression. Int. J. Cancer 2015, 136, 1263-1275. [CrossRef] [PubMed]

4. Lu, W.C.; Kao, S.Y.; Yang, C.C.; Tu, H.F.; Wu, C.H.; Chang, K.W.; Lin, S.C. EGF up-regulates miR-31 through the C/EBPbeta signal cascade in oral carcinoma. PLOS ONE 2014, 9, e108049.

5. Hung, P.S.; Tu, H.F.; Kao, S.Y.; Yang, C.C.; Liu, C.J.; Huang, T.Y.; Chang, K.W.; Lin, S.C. miR-31 is upregulated in oral premalignant epithelium and contributes to the immortalization of normal oral keratinocytes. Carcinogenesis 2014, 35, 1162-1171. [CrossRef] [PubMed]

6. Lu, W.C.; Liu, C.J.; Tu, H.F.; Chung, Y.T.; Yang, C.C.; Kao, S.Y.; Chang, K.W.; Lin, S.C. miR-31 targets ARID1A and enhances the oncogenicity and stemness of head and neck squamous cell carcinoma. Oncotarget 2016, 7, 57254-57267. [CrossRef] [PubMed]

7. Kao, Y.Y.; Chou, C.H.; Yeh, L.Y.; Chen, Y.F.; Chang, K.W.; Liu, C.J.; Fan Chiang, C.Y.; Lin, S.C. MicroRNA miR-31 targets SIRT3 to disrupt mitochondrial activity and increase oxidative stress in oral carcinoma. Cancer Lett. 2019, 456, 40-48. [CrossRef] [PubMed]

8. Lai, Y.H.; Liu, H.; Chiang, W.F.; Chen, T.W.; Chu, L.J.; Yu, J.S.; Chen, S.J.; Chen, H.C.; Tan, B.C. MiR-31-5p-ACOX1 Axis Enhances Tumorigenic Fitness in Oral Squamous Cell Carcinoma Via the Promigratory Prostaglandin E2. Theranostics 2018, 8, 486-504. [CrossRef] [PubMed]

9. Zhu, B.; Cao, X.; Zhang, W.; Pan, G.; Yi, Q.; Zhong, W.; Yan, D. MicroRNA-31-5p enhances the Warburg effect via targeting FIH. FASEB J. 2019, 33, 545-556. [CrossRef]

10. Peng, H.; Hamanaka, R.B.; Katsnelson, J.; Hao, L.L.; Yang, W.; Chandel, N.S.; Lavker, R.M. MicroRNA-31 targets FIH-1 to positively regulate corneal epithelial glycogen metabolism. FASEB J. 2012, 26, 3140-3147. [CrossRef]

11. Lee, M.R.; Mantel, C.; Lee, S.A.; Moon, S.H.; Broxmeyer, H.E. MiR-31/SDHA Axis Regulates Reprogramming Efficiency through Mitochondrial Metabolism. Stem Cell Rep. 2016, 7, 1-10. [CrossRef] [PubMed]

12. Choi, H.Y.; Seok, J.; Kang, G.H.; Lim, K.M.; Cho, S.G. The role of NUMB / NUMB isoforms in cancer stem cells. BMB Rep. 2021, 54, 335-343. [CrossRef] [PubMed]

13. Hwang, W.L.; Jiang, J.K.; Yang, S.H.; Huang, T.S.; Lan, H.Y.; Teng, H.W.; Yang, C.Y.; Tsai, Y.P.; Lin, C.H.; Wang, H.W.; et al. MicroRNA-146a directs the symmetric division of Snail-dominant colorectal cancer stem cells. Nat. Cell Biol. 2014, 16, 268-280. [CrossRef]

14. Li, J.Y.; Huang, W.X.; Zhou, X.; Chen, J.; Li, Z. NUMB inhibits epithelial-mesenchymal transition via RBP-Jkappa-dependent Notch1/PTEN/FAK signaling pathway in tongue cancer. BMC Cancer 2019, 19, 391.

15. Zhong, W.; Jiang, M.M.; Weinmaster, G.; Jan, L.Y.; Jan, Y.N. Differential expression of mammalian NUMB, NUMBlike and Notch1 suggests distinct roles during mouse cortical neurogenesis. Development 1997, 124, 1887-1897. [CrossRef]

16. Flores, A.N.; McDermott, N.; Meunier, A.; Marignol, L. NUMB inhibition of NOTCH signalling as a therapeutic target in prostate cancer. Nat. Rev. Urol. 2014, 11, 499-507. [CrossRef]

17. Di Marcotullio, L.; Ferretti, E.; Greco, A.; De Smaele, E.; Po, A.; Sico, M.A.; Alimandi, M.; Giannini, G.; Maroder, M.; Screpanti, I.; et al. NUMB is a suppressor of Hedgehog signalling and targets Gli1 for Itch-dependent ubiquitination. Nat. Cell Biol. 2006, 8, 1415-1423. [CrossRef]

18. Di Marcotullio, L.; Greco, A.; Mazza, D.; Canettieri, G.; Pietrosanti, L.; Infante, P.; Coni, S.; Moretti, M.; De Smaele, E.; Ferretti, E.; et al. NUMB activates the E3 ligase Itch to control Gli1 function through a novel degradation signal. Oncogene 2011, 30, 65-76. [CrossRef]

19. Carter, S.; Vousden, K.H. A role for NUMB in p53 stabilization. Genome Biol. 2008, 9, 221. [CrossRef] [PubMed]

20. Colaluca, I.N.; Basile, A.; Freiburger, L.; D'Uva, V.; Disalvatore, D.; Vecchi, M.; Confalonieri, S.; Tosoni, D.; Cecatiello, V.; Malabarba, M.G.; et al. A NUMB-Mdm2 fuzzy complex reveals an isoform-specific involvement of NUMB in breast cancer. J. Cell Biol. 2018, 217, 745-762. [CrossRef]

21. Shao, C.; Li, Z.; Ahmad, N.; Liu, X. Regulation of PTEN degradation and NEDD4-1 E3 ligase activity by NUMB. Cell Cycle (Georgetown Tex.) 2017, 16, 957-967. [CrossRef] [PubMed]

22. Gulino, A.; Di Marcotullio, L.; Screpanti, I. The multiple functions of NUMB. Exp. Cell Res. 2010, 316, 900-906. [CrossRef] 
23. Chou, C.H.; Tu, H.F.; Kao, S.Y.; Chiang, C.F.; Liu, C.J.; Chang, K.W.; Lin, S.C. Targeting of miR-31/96/182 to the NUMB gene during head and neck oncogenesis. Head Neck 2018, 40, 808-817. [CrossRef]

24. Peng, H.; Wang, L.; Su, Q.; Yi, K.; Du, J.; Wang, Z. MiR-31-5p promotes the cell growth, migration and invasion of colorectal cancer cells by targeting NUMB. Biomed. Pharmacother. 2019, 109, 208-216. [CrossRef] [PubMed]

25. Hung, P.S.; Liu, C.J.; Chou, C.S.; Kao, S.Y.; Yang, C.C.; Chang, K.W.; Chiu, T.H.; Lin, S.C. miR-146a enhances the oncogenicity of oral carcinoma by concomitant targeting of the IRAK1, TRAF6 and NUMB genes. PLoS ONE 2013, 8, e79926. [CrossRef] [PubMed]

26. Wang, X.; Yang, J.Y.; Cai, J.; Zhang, D.J.; Zhao, L.; Luo, L.H.; Xiong, Y.; Zhang, T.; Jin, M. MiR-543/NUMB promotes proliferation, metastasis, and stem-like cell traits of prostate cancer cells. Am. J. Transl. Res. 2021, 13, 617-631. [PubMed]

27. Li, Y.; Wang, D.; Wang, H.; Huang, X.; Wen, Y.; Wang, B.; Xu, C.; Gao, J.; Liu, J.; Tong, J.; et al. A splicing factor switch controls hematopoietic lineage specification of pluripotent stem cells. EMBO Rep. 2021, 22, e50535. [CrossRef]

28. Wang, N.; Wang, D.D.; Hou, X.; Li, X.; Shen, Y. Different roles of NUMB-p72 and NUMB-p65 on the trafficking of metabotropic glutamate receptor 5. Mol. Biol. Rep. 2021, 48, 595-600. [CrossRef] [PubMed]

29. Chandel, V.; Maru, S.; Kumar, A.; Kumar, A.; Sharma, A.; Rathi, B.; Kumar, D. Role of monocarboxylate transporters in head and neck squamous cell carcinoma. Life Sci. 2021, 279, 119709. [CrossRef]

30. Payen, V.L.; Mina, E.; Van Hee, V.F.; Porporato, P.E.; Sonveaux, P. Monocarboxylate transporters in cancer. Mol. Metab. 2020, 33, 48-66. [CrossRef]

31. Bisetto, S.; Whitaker-Menezes, D.; Wilski, N.A.; Tuluc, M.; Curry, J.; Zhan, T.; Snyder, C.M.; Martinez-Outschoorn, U.E.; Philp, N.J. Monocarboxylate Transporter 4 (MCT4) knockout mice have attenuated 4NQO induced carcinogenesis; A Role for MCT4 in Driving Oral Squamous Cell Cancer. Front. Oncol. 2018, 8, 324. [CrossRef]

32. Zhang, Z.; Gao, Z.; Rajthala, S.; Sapkota, D.; Dongre, H.; Parajuli, H.; Suliman, S.; Das, R.; Li, L.; Bindoff, L.A.; et al. Metabolic reprogramming of normal oral fibroblasts correlated with increased glycolytic metabolism of oral squamous cell carcinoma and precedes their activation into carcinoma associated fibroblasts. Cell Mol. Life Sci. 2020, 77, 1115-1133. [CrossRef] [PubMed]

33. Khammanivong, A.; Saha, J.; Spartz, A.K.; Sorenson, B.S.; Bush, A.G.; Korpela, D.M.; Gopalakrishnan, R.; Jonnalagadda, S.; Mereddy, V.R.; O'Brien, T.D.; et al. A novel MCT1 and MCT4 dual inhibitor reduces mitochondrial metabolism and inhibits tumour growth of feline oral squamous cell carcinoma. Vet. Comp. Oncol. 2020, 18, 324-341. [CrossRef] [PubMed]

34. Wang, Y.; Li, Y.; Jiang, L.; Ren, X.; Cheng, B.; Xia, J. Prognostic value of glycolysis markers in head and neck squamous cell carcinoma: A meta-analysis. Aging (Albany NY) 2021, 13, 7284-7299. [CrossRef]

35. Zhu, J.; Wu, Y.N.; Zhang, W.; Zhang, X.M.; Ding, X.; Li, H.Q.; Geng, M.; Xie, Z.Q.; Wu, H.M. Monocarboxylate transporter 4 facilitates cell proliferation and migration and is associated with poor prognosis in oral squamous cell carcinoma patients. PLoS ONE 2014, 9, e87904. [CrossRef] [PubMed]

36. Khan, A.; Valli, E.; Lam, H.; Scott, D.A.; Murray, J.; Hanssen, K.M.; Eden, G.; Gamble, L.D.; Pandher, R.; Flemming, C.L.; et al. Targeting metabolic activity in high-risk neuroblastoma through Monocarboxylate Transporter 1 (MCT1) inhibition. Oncogene 2020, 39, 3555-3570. [CrossRef]

37. Johansson, A.; Nyberg, W.A.; Sjostrand, M.; Moruzzi, N.; Bergman, P.; Khademi, M.; Andersson, M.; Piehl, F.; Berggren, P.O.; Covacu, R.; et al. miR-31 regulates energy metabolism and is suppressed in T cells from patients with Sjogren's syndrome. Eur. J. Immunol. 2019, 49, 313-322. [CrossRef] [PubMed]

38. Yeh, L.Y.; Yang, C.C.; Wu, H.L.; Kao, S.Y.; Liu, C.J.; Chen, Y.F.; Lin, S.C.; Chang, K.W. The miR-372-ZBTB7A oncogenic axis suppresses TRAIL-R2 associated drug sensitivity in oral carcinoma. Front. Oncol. 2020, 10, 47. [CrossRef] [PubMed]

39. Liu, Z.; Li, H.; Su, J.; Xu, S.; Zhu, F.; Ai, J.; Hu, Z.; Zhou, M.; Tian, J.; Su, Z.; et al. NUMB depletion promotes Drp1-mediated mitochondrial fission and exacerbates mitochondrial fragmentation and dysfunction in acute kidney injury. Antioxid Redox Signal. 2019, 30, 1797-1816. [CrossRef]

40. Gallagher, S.M.; Castorino, J.J.; Philp, N.J. Interaction of monocarboxylate transporter 4 with beta1-integrin and its role in cell migration. Am. J. Physiol. Cell Physiol. 2009, 296, C414-C421. [CrossRef] [PubMed]

41. Slomiany, M.G.; Grass, G.D.; Robertson, A.D.; Yang, X.Y.; Maria, B.L.; Beeson, C.; Toole, B.P. Hyaluronan, CD44, and emmprin regulate lactate efflux and membrane localization of monocarboxylate transporters in human breast carcinoma cells. Cancer Res. 2009, 69, 1293-1301. [CrossRef] [PubMed]

42. Wilson, M.C.; Meredith, D.; Halestrap, A.P. Fluorescence resonance energy transfer studies on the interaction between the lactate transporter MCT1 and CD147 provide information on the topology and stoichiometry of the complex in situ. J. Biol. Chem. 2002, 277, 3666-3672. [CrossRef] [PubMed]

43. Peng, S.Y.; Tu, H.F.; Yang, C.C.; Wu, C.H.; Liu, C.J.; Chang, K.W.; Lin, S.C. miR-134 targets PDCD7 to reduce E-cadherin expression and enhance oral cancer progression. Int. J. Cancer 2018, 143, 2892-2904. [CrossRef]

44. Lin, S.C.; Wu, H.L.; Yeh, L.Y.; Yang, C.C.; Kao, S.Y.; Chang, K.W. Activation of the miR-371/372/373 miRNA cluster enhances oncogenicity and drug resistance in oral carcinoma cells. Int. J. Mol. Sci. 2020, 21, 9442. [CrossRef] [PubMed] 